\title{
Dual Entropy Regime in Channel Flow Subjected to Temperature Dependent Convection Mechanism
}

\author{
Paresh Vyas*, Sahanawaz Khan \\ Department of Mathematics, University of Rajasthan, Jaipur 302004, India
}

Corresponding Author Email: sahanawazk201@gmail.com

https://doi.org/10.18280/ijht.390211

Received: 21 September 2020

Accepted: 10 January 2021

\section{Keywords:}

entropy, radiation parameter, porous medium, temperature dependent convection mechanism

\begin{abstract}
The central stimuli of this brief note is to underscore the effect of the temperature dependent convection coefficient that give rise to a dual temperature regime facilitating dual entropy distribution. In order to avoid unwarranted complexities, a simple geometry of shear flow in a channel is considered. The energy equation amenable to an analytic solution is simulated to extract the desired numerical findings in as much as for what parameters' values, the temperature has dual distribution /does not yield temperature distribution at all. In fact, a range of parameter values have been worked out for which dual temperature regime exists or not. The plots of entropy generation number Ns also show the dual regime. The findings reveal a qualitative and quantitative difference in dual systems of temperature and entropy. It further underlines that the thermal systems with the idealized uniform heat transfer coefficient may be far distinct from actual behaviour and even weak temperature dependence of convection coefficient need due attention while designing a system.
\end{abstract}

\section{INTRODUCTION}

Simulating thermo-fluidic systems for entropy aspects has had started gaining currency in the last couple of decades. The literature reveals that the studies were prompted to devise cost effective strategies for efficient energy usages. Heat transfer and/or entropy aspects in channel flow with or without porous inserts have drawn attention of the investigators. Fluid flows involving porous-inserts- geometries find a parallel in biofluid transport, heat exchangers, thermal insulation engineering, cooling of electronic components, filtration process etc.

Chauhan and Vyas [1] reported heat transfer in compressible Couette fluid flow in a permeable channel. Mahmud and Fraser [2] considered non- Newtonian fluid flow in channels/pipe with an objective to investigate thermodynamic irreversibility. Yilbas et al. [3] conducted an entropy analysis for non-Newtonian fluid flow in annular pipe. Haddad et al. [4] analyzed the entropy generation forced convection flow in a micro channel. Mahmud and Fraser [5] discussed thermal characteristics and entropy generation inside a dissipative porous channel. Hooman and Ejlali [6] considered forced convection in a saturated porous circular tube to study entropy generation. Chen and Zhu [7] chose Bingham fluid to study Couette-Poiseuille flow between porous parallel plates with slip conditions. They showed that the slippage weakens the shearing deformation. Eegunjobi and Makinde [8] considered a flow in a porous channel to examine the effects of Navier slip on entropy generation when the system was subjected to suction/injection.

Vyas and his co-authors [9-15] investigated entropy generation in various thermo-fluidic configurations. Chinyoka and Mankinde [16] presented an entropy generation analysis for an unsteady channel flow. Yazdi et al. [17] reported findings on the entropy in specifically arranged micro channels embedded in a permeable surface. Ibanez et al. [18] conducted a study on minimization of entropy in micro channels. Makinde and Eegunjobi [19] simulated convective heating effects on entropy generation for the flow inside a permeable channel. Vyas and Srivastava [20] also examined radiative MHD Couette flow of compressible fluid in a channel with permeable base. Ibanez et al. [21] investigated the effects of heat flux and velocity slip on entropy generation in a micro channel. Lopez et al. [22] studied entropy generation of power-law fluid in a channel with asymmetric convective cooling. Ibanez [23] investigated entropy generation in the MHD porous channel with hydrodynamic slip and convective boundary conditions. Srinivasacharya and Bindu [24] considered micropolar fluid flow in an inclined channel for entropy studies. Kareem et al. [25] simulated entropy generation for MHD couple-stress fluid flow in a porous channel. Ajibade and Onoja [26] reported an irreversibility analysis for steady mixed convection in a vertical channel. thermodynamic irreversibility in thermofluidic systems for various features such as nano fluid, radiating fluid, microchannel etc. were taken up in refs. [2736].

The said flow geometries and many others not reported here have been treated for cases where the system is subjected to a heat flux with uniform heat transfer coefficient. However, the idealized thermal systems with uniform heat transfer coefficient may not always be at par with similarly situated real systems simply because the heat convection mechanism may be significantly temperature dependent. In fact, in certain applications, the heat transfer may show up qualitative and quantitative changes with local temperature e.g. the cooling process may follow a power law type temperature dependence or it experiences "weakly" linear temperature dependence. By 
physical understanding of convective mechanism, there is no debate over the fact that the convection heat transfer rate is proportional to convection heat transfer coefficient. There are some pertinent studies shedding light on temperature dependent convection mechanism. Ghai and Jacob [37] presented a pertinent text on local coefficient of heat transfer on fins. Lau and Tan [38] reported errors in one-dimensional heat transfer analysis for the straight and annular fins. Laor and Kalman [39] discussed the effects of heat transfer coefficient which is temperature dependent on performance of cooling fins. Unal [40] analyzed one-dimensional longitudinal fins considering the case where the heat transfer coefficient is assumed to follow a power law relation of the fin and fluid temperatures. He extracted a temperature distribution for an extended surface subjected to a non-uniform heat transfer coefficient. Sen and Trinh [41] derived an exact solution for the heat transfer rate for a rectangular fin with power law-type temperature dependence of convection coefficient. Vyas and Ranjan [42] examined forced convection with temperature dependent convection mechanism and found a unique temperature distribution.

In this paper, we report a dual temperature distribution for a forced convection flow in the permeable channel. The central stimuli of the paper are to underscore that how a convection mechanism with even "weakly" temperature dependent convection coefficient give rise to dual temperature regime and consequently facilitates a dual entropy distribution system. A range of parameter values have been worked out for which the said dual system exists or not, and their effects have been investigated.

\section{MATHEMATICAL FORMULATION}

Let us consider a steady radiative MHD Couette shear flow in a permeable channel. The channel comprises of two parallel walls distant $\mathrm{H}$ apart. The lower stationary wall is naturally permeable and is subjected to a convective flux with "weakly" temperature dependent convective coefficient and a hydrodynamic slip. The impermeable upper wall is assumed to move in its own plane with a uniform velocity $\mathrm{u}_{0}$ and bears a uniform temperature $\mathrm{T}_{0}$. A Cartesian coordinate system is considered as shown in the schematic diagram (Figure 1). A magnetic field of strength $B_{0}$ is applied transverse to the flow direction. The induced magnetic field is assumed to be negligible. Beavers and Joseph [43] conducted experiments for flow in a channel with porous base. They found a velocity slip at the clear fluid- porous interface revealing that the shear effects were propagated into the porous strata through a boundary layer region and the effect could be conceived as a hydrodynamic slip condition at the fluid-porous medium interface. Furthermore, though the slug flow in the permeable bed addressed by Darcy's model, but in the absence of any external pressure gradient and for small permeability, the interior flow of the porous medium would not contribute much to the exterior clear fluid flow, and therefore, zero filter velocity in the permeable bed may be assumed. However, the permeability of the lower bed affects the clear fluid flow through the macroscopic boundary condition (that accounts for the hydrodynamic slip) as suggested by Saffman [44]. By this condition under the present setup the following equation is appropriate to compute the exterior clear fluid flow correct to $\mathrm{O}(\mathrm{K})$.

$$
u^{*}=\frac{\sqrt{K^{*}}}{\alpha}\left(\frac{d u^{*}}{d y^{*}}\right)+O\left(K^{*}\right)
$$

where, $u^{*}$ is the tangential fluid velocity, $K^{*}$ is the permeability, $\alpha$ is the dimensionless empirical constant and $y$ is the direction normal to the boundary. The constant $\alpha$ depends the structure of the permeable material within the boundary region. The above condition put forward by Saffman was also got validated by Dagan [45]. It is not out of place to put on record that Beavers and Joseph [43] computed values for $\alpha$ as 0.78 , and 4.0 for foametal having average pore sizes 0.016 and 0.045 inches, respectively, and further 0.1 for aloxite with average pore size 0.027 inches.

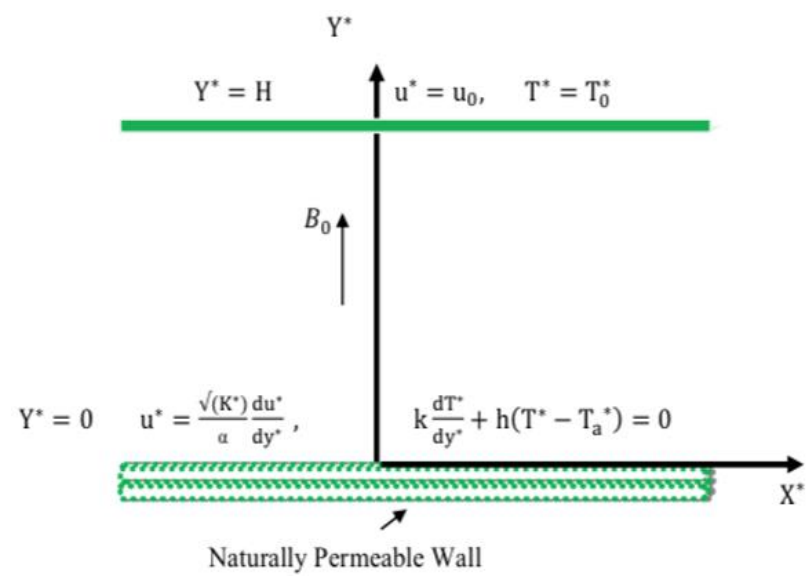

Figure 1. Schematic diagram - mathematical model

For more on the said condition and allied discussion/configurations, interested readers may refer to Chauhan and Vyas [1], Vyas and Srivastava [20] and the references [43-50].

Under said assumptions, the governing equations and the end conditions are as follows:

$$
\begin{gathered}
\mu\left(\frac{d^{2} u^{*}}{d y^{* 2}}\right)-\sigma B_{0}^{2} u^{*}=0 \\
k\left(\frac{d^{2} T^{*}}{d y^{* 2}}\right)+\sigma B_{0}^{2} u^{* 2}+\mu\left(\frac{d u^{*}}{d y^{*}}\right)^{2}-\frac{d q_{r}}{d y^{*}}=0
\end{gathered}
$$

And the boundary conditions are:

$$
\left\{\begin{array}{l}
y^{*}=0 ; \quad u^{*}=\frac{\sqrt{K^{*}}}{\alpha} \frac{d u^{*}}{d y^{*}} ; \quad \mathrm{k} \frac{d T^{*}}{d y^{*}}+h\left(T^{*}-T_{a}^{*}\right)=0 \\
y^{*}=H ; \quad u^{*}=u_{0}^{*} ; \quad \mathrm{T}^{*}=T_{0}^{*}
\end{array}\right\}
$$

where, $u^{*}$ denotes fluid velocity, $\mu$ is the viscosity, $\mathrm{k}$ is thermal conductivity, $K^{*}$ permeability, $\sigma$ electric conductivity, $\mathrm{h}$ temperature dependent convective coefficient, $T^{*}$ fluid temperature, $T_{a}^{*}$ is the ambient temperature, $\alpha$ slip coefficient and $q_{r}$ is radiation flux.

We assume that the convection coefficient " $h$ " bears a weakly temperature dependence of the form. 


$$
h=h_{a}\left\{1+\varepsilon\left(\frac{T^{*}-T_{a}^{*}}{T_{a}^{*}}\right)\right\}
$$

where, $h_{a}$ is the reference convection coefficient and $\varepsilon$ is the small linearity constant. The radiation flux $q_{r}$ follows the Rosseland approximation and is given by:

$$
q_{r}=-\frac{4 \gamma^{*}}{3 \delta^{*}} \frac{d T^{* 4}}{d y^{*}}
$$

where, $\gamma^{*}$ and $\delta^{*}$ being Stephen Boltzmann constant and Mean absorption constant respectively. Further, we approximate $T^{* 4}$ as a linear function of $T^{*}$ as follows:

$$
T^{* 4} \cong 4 T_{0}^{* 3} T^{*}-3 T_{0}^{* 4}
$$

The above linearization of the temperature $T^{*}$ obtained by a truncated Taylor series expansion of $T^{*}$ about $T_{0}^{*}$ is valid when the temperature difference in the fluid is small enough.

We introduce the following non-dimensional quantities as:

$$
\eta=\frac{y^{*}}{H}, \quad u=\frac{u^{*}}{u_{0}}, \quad \theta=\frac{\left(T^{*}-T_{a}^{*}\right)}{T_{0}^{*}}, \quad K=\frac{K^{*}}{H^{2}}
$$

The Eqns. (2) and (3) becomes:

$$
\begin{gathered}
\frac{d^{2} u}{d \eta^{2}}-M^{2} u=0 \\
(1+N) \frac{d^{2} \theta}{d \eta^{2}}+B r\left\{M^{2} u^{2}+\left(\frac{d u}{d \eta}\right)^{2}\right\}=0
\end{gathered}
$$

And the boundary condition (4)

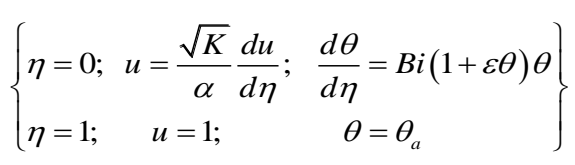

where, $M=\sqrt{\left(\frac{\sigma B_{0}^{2} H^{2}}{\mu}\right)}, B r=\frac{\mu u_{0}^{2}}{k T_{a}}$ and $N=\frac{16 \gamma^{*} T_{0}^{3}}{3 \delta^{*} k}$ are denotes as Hartmann number, Brinkmann number and radiation parameter respectively and $\theta_{a}=\frac{\left(T_{0}-T_{a}\right)}{T_{a}}=\omega-1$ and $B i=-\frac{h_{a} H}{k}$ are temperature ratio and Biot Number.

The local volumetric rate of entropy generation $S_{G}$ for the configuration under study is found as (following the authors in $[9,12,13,20,25,33,34])$

$$
S_{G}=\frac{k}{T_{0}^{* 2}}(1+N)\left(\frac{d T^{*}}{d Y^{*}}\right)^{2}+\frac{\mu}{T_{0}^{* 2}}\left(\frac{d u^{*}}{d y^{*}}\right)^{2}+\sigma \frac{B_{0}^{2} u^{*^{2}}}{T_{0}^{*^{2}}}
$$

We prescribe,
$S_{G_{0}}=\frac{k}{H^{2}} \frac{T_{a}^{*^{2}}}{T_{0}^{*^{2}}}$ (The characteristic entropy generation rate).

$\omega=\frac{T_{0}^{*}}{T_{a}^{*}}$ (The characteristic temperature ratio).

Thus, the non-dimensional entropy generation number $N_{s}$ is obtained as follows:

$$
N_{s}=\frac{S_{G}}{S_{G_{0}}}=(1+N)\left(\frac{d \theta}{d \eta}\right)^{2}+B r \omega\left\{\left(\frac{d u}{d \eta}\right)^{2}+M^{2} u^{2}\right\}
$$

\section{SOLUTION}

As evident from the Eq. (12), we require velocity and thermal temperature distributions and their gradients to compute the entropy generation number. The solution of (8) and (9) is straightforward is found as follows:

$$
u=C_{1} e^{M \eta}+C_{2} e^{-M \eta}
$$

$$
\theta=\frac{-B r}{2(1+N)}\left(C_{1}^{2} e^{2 M \eta}+C_{2}^{2} e^{-2 M \eta}\right)+D_{1} \eta+D_{2}
$$

where, the constants of integration $C_{1}, C_{2}, D_{1}$ and $D_{2}$ are as follows:

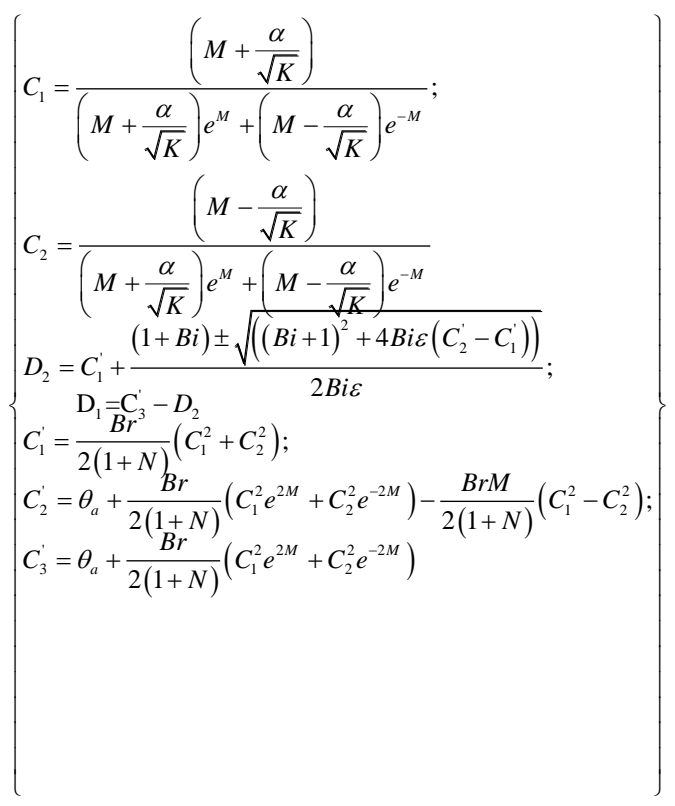

Thus, the entropy generation number $N_{s}$ is obtained as follows

$$
\begin{aligned}
& N_{s}=(1+N)\left\{\frac{-B r M}{(1+N)}\left(C_{1}^{2} e^{2 M \eta}-C_{2}^{2} e^{-2 M \eta}\right)+D_{1}\right\}^{2} \\
& +2 B r \omega M^{2}\left(C_{1}^{2} e^{2 M \eta}-C_{2}^{2} e^{-2 M \eta}\right)
\end{aligned}
$$

\section{RESULT AND DISCUSSION}

The problem in hand is peculiar in the sense that the for certain sets of parameter values, the energy Eq. (10) yields 
either two distinct real solutions or no real solution. we have a real dual solution regime for the temperature as evident from the plots reported here. Furthermore, the dual solution for the energy equation leads to a dual entropy distribution too.

The reason unfolds if we look at the constants of integration. We find that the constant $D_{2}$ depends on the constants $C_{1}$ and $C_{2}$ which involve other parameters entering into the problem. Keeping other parameters fixed, we worked out a range of Biot number $\mathrm{Bi}$ for which the expression $\left((B i+1)^{2}+4 B i \varepsilon\left(C_{2}^{\prime}-C_{1}^{\prime}\right)\right)$ appearing in constant $D_{2}$ is the negative i.e. the case for which there is no real solution. We found many combinations of parameters' values that led to a dual solution or no real solution for the energy equation.

The Tables 1-5 presented here to showcase the range of a few sets of parameter values for which no solution exists. To be specific, the Table 1 presents two pertinent information for given $\varepsilon$ and other parameters fixed, the interval of $\mathrm{Bi}$ values for which there is no real solution and vice versa i.e. for given $\mathrm{Bi}$, the interval of $\varepsilon$ values for which the solution does not exist. Similarly, the other tables present the respective range of parameter values for which real solution for the energy equation does not exist.

From the Table 1 we see that (for fixed values $\alpha=0.1$, $\left.\mathrm{K}=0.001, \mathrm{M}=1, \mathrm{Br}=40, \mathrm{~N}=1, \theta_{\mathrm{a}}=-0.2, \omega=0.8\right)$ when $\varepsilon=0.001$ is changed to $\varepsilon=0.1$ then the range of $\mathrm{Bi}$ values for which no solution exists changes from $(-0.8447,-1.1841)$ to $(-0.2153$, 4.6456). This is worth noting that how the system may be significantly sensitive to the choice of the linearity constant. Furthermore, the Table also shows the range of $\varepsilon$ values for given $\mathrm{Bi}$. We note that when $\mathrm{Bi}=-2$, then there is no solution for $\varepsilon \in(0.0174, \infty)$ and the range for $\varepsilon$ hanges to $(0.1118, \infty)$ when $\mathrm{Bi}=-5$. Similarly, other Tables present select computations of other parameter values for which energy equation does not yield real distribution.

Table 1. The range of Biot number Bi and linearity constant $\varepsilon$ for which the solution does not exist when $\alpha=0.1, K=0.001, M=1$, $B r=40, N=1, \theta_{\mathrm{a}}=-0.2, \omega=0.8$

\begin{tabular}{cccc}
\hline Epsilon $(\boldsymbol{\varepsilon})$ & Range of Biot Number (Bi) & Biot Number $(\mathbf{B i})$ & Range of Epsilon $(\varepsilon)$ \\
\hline 0.001 & $(-0.8447,-1.1841)$ & -2 & $(0.0174, \infty)$ \\
0.01 & $(-0.5893,-1.6967)$ & -3 & $(0.0466, \infty)$ \\
0.05 & $(-0.3218,-3.1087)$ & -4 & $(0.0786, \infty)$ \\
0.1 & $(-0.2153,-4.6456)$ & -5 & $(0.1118, \infty)$ \\
\hline
\end{tabular}

Table 2. The range of Biot number $B i$ and Hartmann number $M$ for which the solution does not exist when $\alpha=0.1, K=0.001$, $\varepsilon=0.1, B r=40, N=1, \theta_{\mathrm{a}}=-0.2, \omega=0.8$

\begin{tabular}{cccc}
\hline Hartmann Number (M) & Range of Biot Number (Bi) & Biot Number (Bi) & Range of Hartmann Number (M) \\
\hline 1 & $(-0.2153,-4.6465)$ & -1 & $(0.00000001, \infty)$ \\
2 & $(-0.1848,-5.4103)$ & -2 & $(0.00000001, \infty)$ \\
3 & $(-0.1765,-5.6749)$ & -3 & $(0.00000001, \infty)$ \\
& & -4 & $(0.15, \infty)$ \\
& & -5 & $(1.37995, \infty)$ \\
\hline
\end{tabular}

Table 3. The range of Biot number Bi and Brinkmann number $\mathrm{Br}$ for which the solution does not exist when $\alpha=0.1, K=0.001$, $\mathrm{M}=1, \varepsilon=0.1, N=1, \theta_{\mathrm{a}}=-0.2, \omega=0.8$

\begin{tabular}{cccc}
\hline Brinkmann Number (Br) & Range of Biot Number (Bi) & Biot Number (Bi) & Range of Brinkmann Number (Br) \\
\hline 30 & $(-0.2587,-3.8670)$ & -1 & $(1.0881, \infty)$ \\
40 & $(-0.2153,-4.6456)$ & -2 & $(7.8889, \infty)$ \\
50 & $(-0.1849,-5.4112)$ & -3 & $(19.2237, \infty)$ \\
\hline
\end{tabular}

Table 4. The range of Biot number Bi and Radiation parameter $N$ for which the solution does not exist when $\alpha=0.1, K=0.001$, $M=1, \varepsilon=0.1, B r=40, \theta_{a}=-0.2, \omega=0.8$

\begin{tabular}{cccc}
\hline Radiation Parameter (N) & Range of Biot Number (Bi) & Biot Number (Bi) & Range of Radiation Parameter (N) \\
\hline 1 & $(-0.2153,-4.6456)$ & -2 & $(0,9.1407)$ \\
2 & $(-0.2775,-3.6029)$ & -3 & $(0,3.1615)$ \\
3 & $(-0.3264,-3.0640)$ & -4 & $(0,1.5244)$ \\
\hline
\end{tabular}

Table 5. The range of Biot number Bi and Brinkmann number $\mathrm{Br}$ for which the solution does not exist when $\alpha=0.1, K=0.001$, $M=1, \varepsilon=0.1, N=1, \theta_{a}=-0.2, \omega=0.8$

\begin{tabular}{cccc}
\hline Brinkmann Number (Br) & Range of Biot Number (Bi) & Biot Number (Bi) & Range of Hartmann Number (Br) \\
\hline 30 & $(-0.2476,-3.8670)$ & -1 & $(0, \infty)$ \\
40 & $(-0.2077,-4.8130)$ & -2 & $(5.7128, \infty)$ \\
50 & $(-0.1793,-5.5764)$ & -3 & $(17.0475, \infty)$ \\
\hline
\end{tabular}




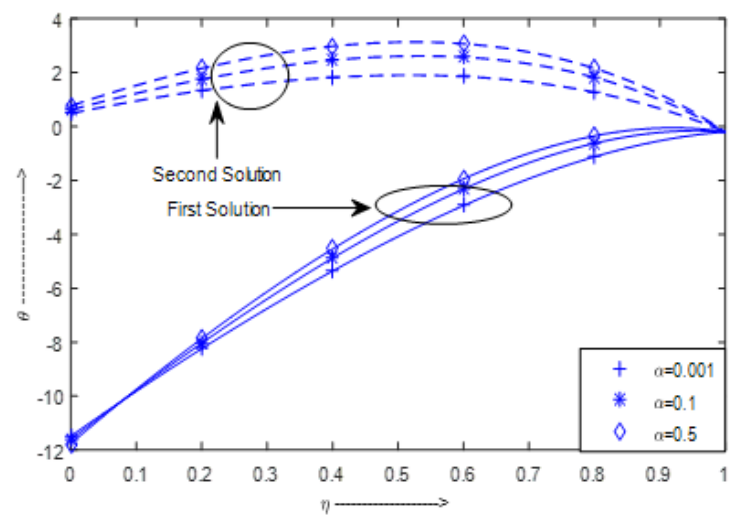

Figure 2. Variation in temperature as varying $\alpha$ or $K=0.001$, $M=1, \varepsilon=0.1, B r=40, N=1, B i=10, \theta_{\mathrm{a}}=-0.2, \omega=0.8$

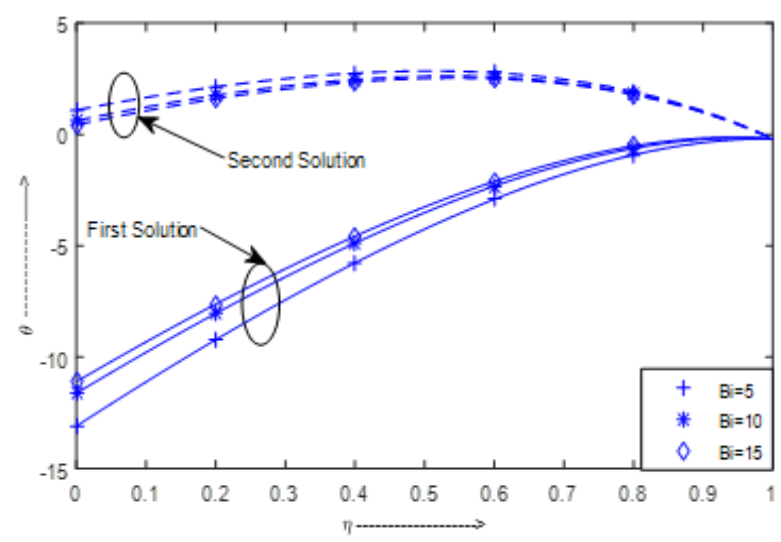

Figure 3. Variation in temperature as varying $B i$ for $\alpha=0.1$, $K=0.001, M=1, \varepsilon=0.1, B r=40, N=1, \theta_{\mathrm{a}}=-0.2, \omega=0.8$

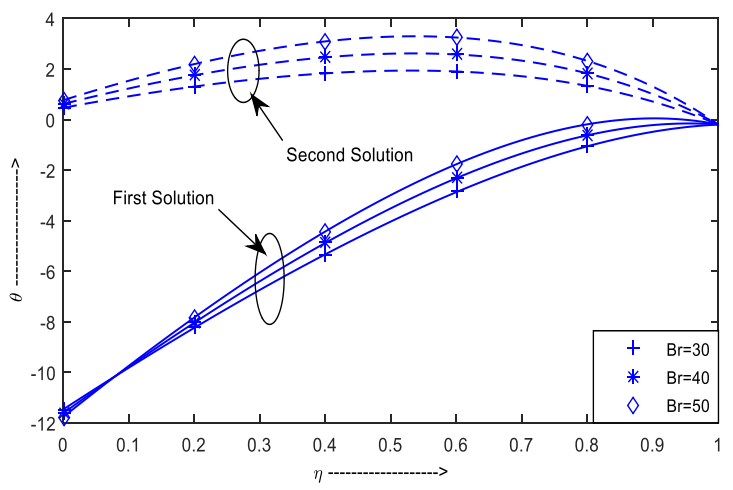

Figure 4. Variation in temperature as varying $B r$ for $\alpha=0.1$, $K=0.001, M=1, \varepsilon=0.1, N=1, B i=10, \theta_{\mathrm{a}}=-0.2, \omega=0.8$

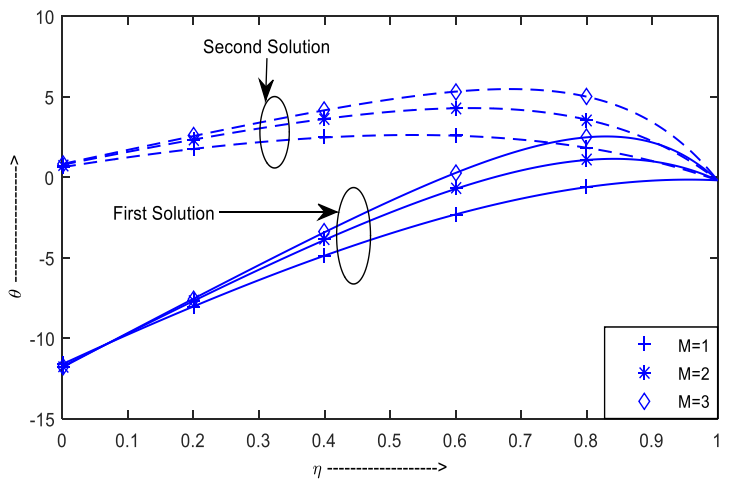

Figure 5. Variation in temperature as varying $M$ for $\alpha=0.1$, $K=0.001, \varepsilon=0.1, B r=40, B i=10, \mathrm{~N}=1, \theta_{\mathrm{a}}=-0.2, \omega=0.8$

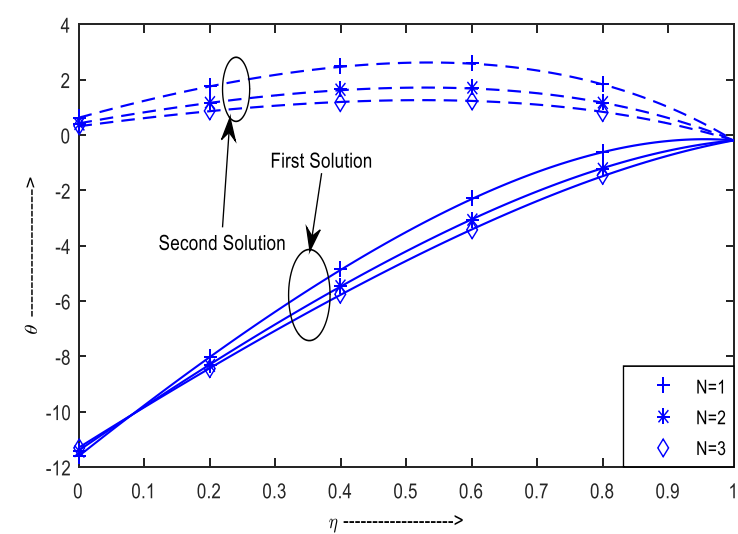

Figure 6. Variation in temperature as varying $N$ for $\alpha=0.1$, $K=0.001, \mathrm{M}=1, \varepsilon=0.1, B r=40, B i=10, \theta_{\mathrm{a}}=-0.2, \omega=0.8$

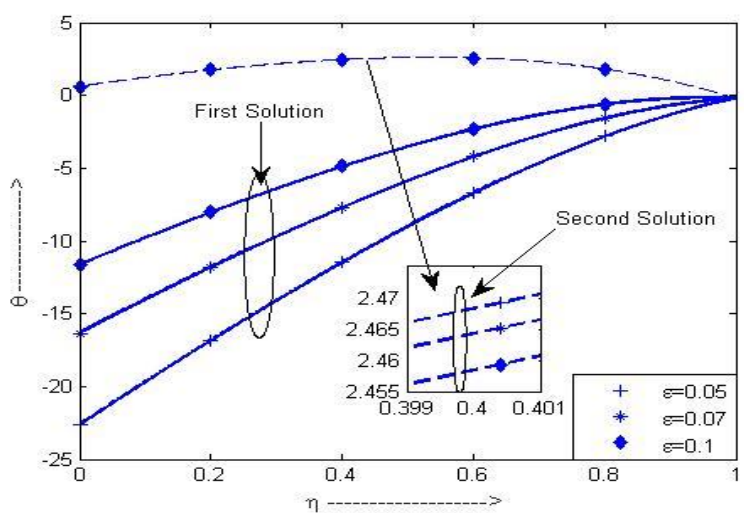

Figure 7. Variation in temperature as varying $\varepsilon$ for $\alpha=0.1$, $K=0.001, M=1, B r=40, B i=10, N=1 \theta_{\mathrm{a}}=-0.2, \omega=0.8$

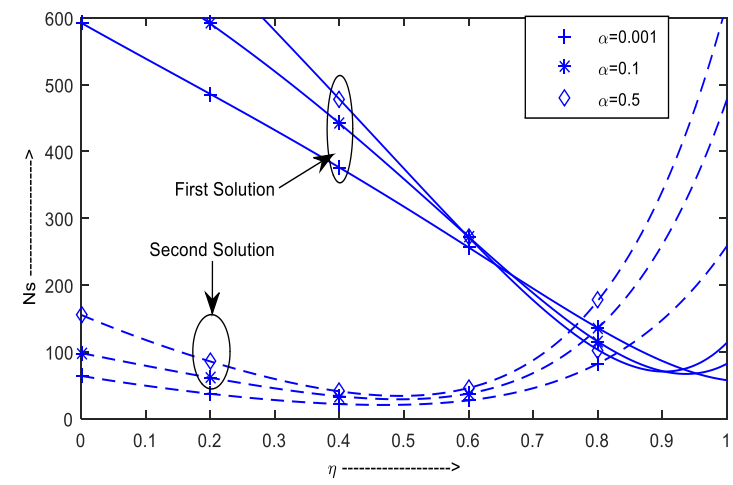

Figure 8. Variation in entropy generation number $N_{s}$ for varying $\alpha$ for $K=0.001, M=1, \varepsilon=0.1, B r=40, N=1, B i=10$, $\theta_{\mathrm{a}}=-0.2, \omega=0.8$

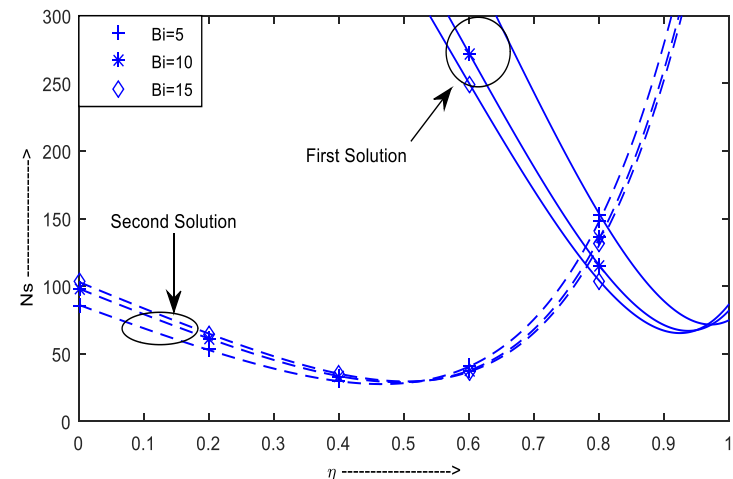

Figure 9. Variation in entropy generation number $N_{s}$ for varying $B$ i for $\alpha=0.1, K=0.001, M=1 \varepsilon=0.1, B r=40, N=1$, $\theta_{\mathrm{a}}=-0.2, \omega=0.8$ 


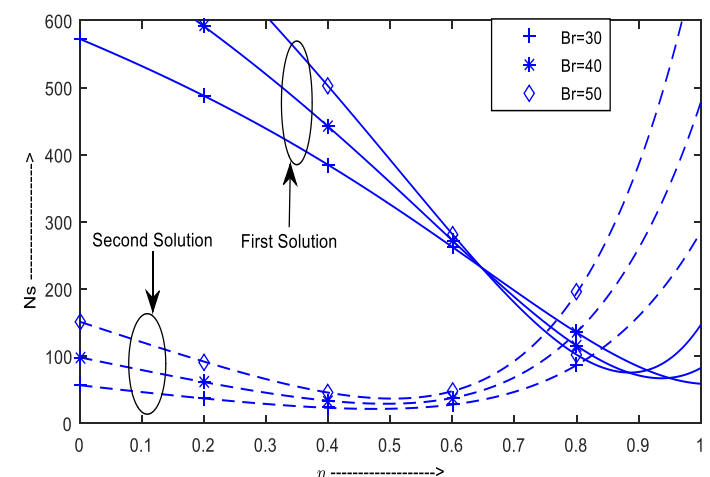

Figure 10. Variation in entropy generation number $N_{s}$ for varying $B r$ for $\alpha=0.1, K=0.001, M=1 \varepsilon=0.1, N=1, B r=10$, $\theta_{\mathrm{a}}=-0.2, \omega=0.8$

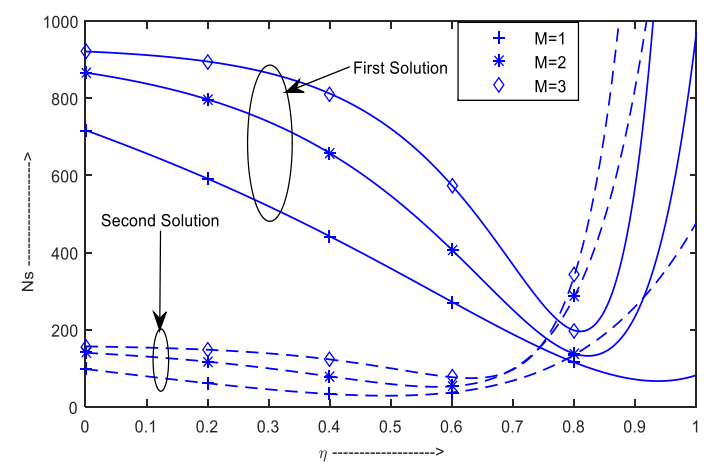

Figure 11. Variation in entropy generation number $N_{s}$ for varying $M$ for $\alpha=0.1, K=0.001, \varepsilon=0.1, B r=40, B i=10, N=1$, $\theta_{\mathrm{a}}=-0.2, \omega=0.8$

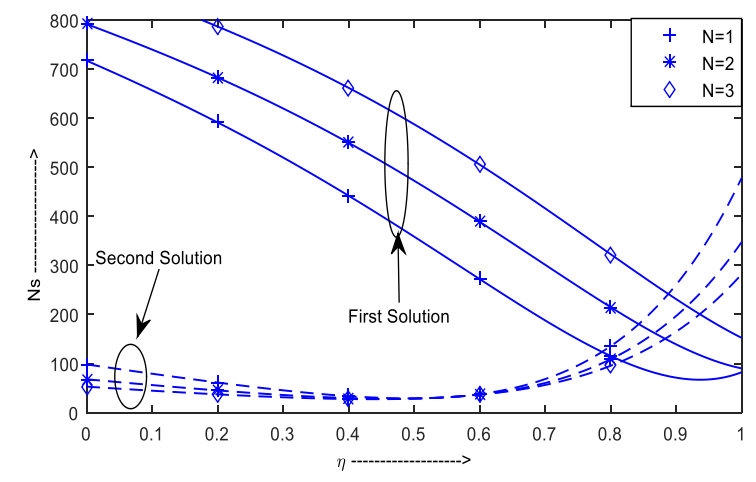

Figure 12. Variation in entropy generation number $N_{s}$ for varying $N$ for $\alpha=0.1, K=0.001, M=1 \varepsilon=0.1, B r=40, B i=10$, $\theta_{\mathrm{a}}=-0.2, \omega=0.8$

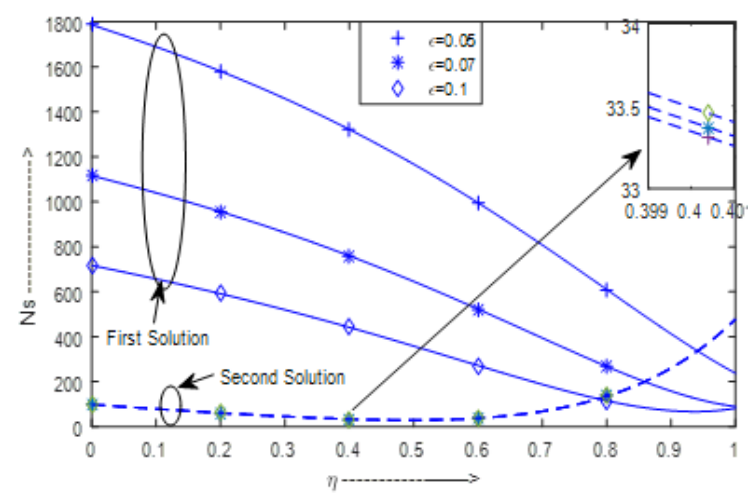

Figure 13. Variation in entropy generation number $N_{s}$ for varying $\varepsilon$ for $\alpha=0.1, K=0.001, M=1, B r=40, B i=10, N=1$, $\theta_{\mathrm{a}}=-0.2, \omega=0.8$
The Figures 2-7 depict temperature distributions. Figures 813 portray the local entropy generation number Ns. By these figures, we see that for one set of values of parameters there are two real distinct temperature regimes and consequently dual plots to the entropy are also obtained. In order to distinguish the two, we term these "first" and "second" solutions. The deciphering of graphs and their physical utility is a pertinent question and the information may be a useful basis for the intricacies required to create a system of interest where entropy generation analysis is instrumental for entropy generation minimization. This understanding gets weight in view of qualitative and quantitative differences observed in the first and second solution for temperature and entropy distributions. Plots for the temperature and entropy clearly show that the system is quite sensitive to the combination of parameter values and the phenomenon can be treated or discussed case by case. However, if we concentrate on the cases reported here then we see that the first and second distributions of temperature are the prominently distinct and exhibit peculiar pattern for varying values of the parameters. The Figure 2 shows the effect of slip coefficient $\alpha$ on the temperature. The effect is strikingly visible on both first and second temperature distributions. The Figure 3 depicts that the effect of $\mathrm{Bi}$ on first and second solutions (temperature distributions) is not similar. We see that in the case of first temperature distribution, the temperature shows an increase for increasing values of Biot number. However, for the second temperature distribution there is an opposite trend. Figure 4 presents a different story that for increasing values of Brinkman number $\mathrm{Br}$ we see that the temperature increases in the first as well as in the second distribution. The Figure 5 also exhibits the same trend for the increasing values of Hartmann number as we have noted in the Figure 4, except in a region (spatial distance $0<\eta<0.15$ ) for the first distribution. The same trend is also witnessed for both the first and second distribution in the case of varying radiation parameter (Figure 6 ). The Figure 7 reveals the effect of the linearity constant $\varepsilon$ on the temperature. It is found that a change in $\varepsilon$ causes a qualitative change in the first temperature distribution in contrast to impact on the second distribution. The Figures 813 depicting variations in Ns clearly manifest that the entropy generation has a defining different spatial distribution viz. first and the second as we would term them. One can observe a marked quantitative difference between the two entropy distributions arising out of the dual temperature regimes for a variety of the sets of parameter values. The question remains to be answered as to how the dual system may be harnessed for better understanding for devising tool and /or designing tool.

\section{CONCLUSIONS}

In this paper, a steady radiative MHD Couette shear flow of an incompressible fluid in a parallel wall channel was considered. The naturally permeable bottom of the channel was subjected to a convective flux with "weakly" temperature dependent convective coefficient and a hydrodynamic slip. A magnetic field of uniform strength was applied transverse to the flow direction. The model solved analytically showed that "weakly" temperature dependence of convection coefficient resulted in a peculiar situation for energy equation. It was found that the equation admitted either two distinct real solutions or no real solution for select parameter' values. 
Furthermore, the temperature dependent weak perturbations in convection coefficient not only affected temperature distribution but also reflected in entropy distribution too. The dual solution for the energy equation led to a dual entropy distribution too.

The findings portrayed through tables and the graphs unequivocally transpire that how even a weakly temperature dependent convective mechanism may be altogether different from a convective mechanism with uniform convection coefficient which have unique temperature profiles system in latter case. The most intriguing question here was to highlight that convection mechanism sensitive/ prone to temperature dependence needed due care while devising a system where the thermal characteristics may be a prime concern. The paper computed range of parameter (when others are kept fixed) for which no real solution exists / dual solution exists to the energy equation. The mathematical simulation conducted here, in our opinion, would lead to revisit of many convective mechanisms. The key observations are:

1. The simulation gave rise to a wide range of values of parameters for which either no real unique temperature distribution or a dual temperature distribution exists. An extensive computation has been done to find the range of a few sets of parameter values for which no real solution of the energy equation exists. The tables show case the situations for what vales of parameters solution does not exist at all. For example, when $\alpha=0.1, K=0.001, M=1, B r=40, N=1, \theta_{a}=-0.2$, $\omega=0.8$ and $\varepsilon=0.001$ is changed to $\varepsilon=0.1$, then the range of $\mathrm{Bi}$ values for which no solution exists changes from $(-0.8447$, $1.1841)$ to $(-0.2153,4.6456)$. This highlights that how the system may be significantly sensitive to the choice of the linearity constant i.e. perturbation in convection coefficient. Furthermore, when $\mathrm{Bi}=-2$, then there is no solution for $\varepsilon \in(0.0174, \infty)$ and the range for $\varepsilon$ changes to $(0.1118, \infty)$ the text present regorous computations for parametric studies to peep into the issue.

2. The simulation revealed that in the cases of dual solution for the energy equation, both the solutions are qualitatively and quantitatively distinct. In fluid mechanics, there are thermal configurations that admit multiple solutions for momentum and energy equations such as boundary layer flow over a shrinking sheet. the multiplicity of solutions is found due to suction rates. Here in our case study, the "onus" for the multiplicity of the solution is found on the perturbation in convection coefficient.

3 . The configuration undertaken here is rather simpler in terms of geometry and can be handled with simple mathematical modelling. However, the presented baby model has a scope for future explorations with rather more complexities e. g. strong temperature dependent convection mechanism, nonuniform physical properties of fluid, compressibility etc. In this paper, we excused ourselves from treading on these for the simple reason that we first intended to show pertinent shift arising out of weak perturbations in convection coefficient.

\section{REFERENCES}

[1] Chauhan, D.S., Vyas, P. (1995). Heat transfer in hydromagnetic Couette flow of compressible Newtonian fluid. ASCE Journal of Engineering Mechanics, 121(1): 57-61. https://doi.org/10.1061/(ASCE)07339399(1995)121:1(57)
[2] Mahmud, S., Freser, R.A. (2002). Inherent irreversibility of channel and pipe blows for non-Newtonian fluids. Int. Commn. Heat Mass Transfer, 29(5): 577-587. https://doi.org/10.1016/S0735-1933(02)00377-9

[3] Yilbas, B.S., Yursoy, M., Pakdemerili, M. (2004). Entropy analysis for non-Newtonian fluid flow in annular pipe: constant viscosity case. Entropy, 6(3): 304315. https://doi.org/10.3390/e6030304

[4] Haddad, O., Abuzaid, M., Al-Nimr, M. (2004). Entropy generation due to laminar incompressible forced convection flow through parallel plates micro channel. Entropy, 6(5): 413-426. https://doi.org/10.3390/e6050413

[5] Mahmud, S., Fraser, R.A. (2005). Flow, thermal and entropy generation characteristics inside a porous channel with viscous dissipation. Int. J. Thermal Science, 44(1): 21-32. https://doi.org/10.1016/j.ijthermalsci.2004.05.001

[6] Hooman, K., Ejlali, A. (2007). Entropy generation for forced convection in a porous saturated circular tube with uniform wall temperature. Int. Commn. Heat Mass Transfer, 34(4): 408-419. https://doi.org/10.1016/j.icheatmasstransfer.2006.10.00 8

[7] Chen, Y., Zhu, K. (2008). Coquette-Poisieille flow of Bingham fluids between two porous parallel plates with slip conditions. J. Non-Newtonian Fluid Mech., 153(1): 1-11. https://doi.org/10.1016/j.jnnfm.2007.11.004

[8] Eegunjobi, A.S., Makinde, O.D. (2012). Effects of Navier slip on entropy generation in a porous channel with suction/injection. J. Therm. Sci., 7(4): 522-535. https://doi.org/10.1299/jtst.7.522

[9] Vyas, P., Kasana, R.K., Khan, S. (2020). Entropy Analysis for boundary layer Micropolar fluid flow. AIMS Mathematics, 5(3): 2009-2026. https://doi.org/10.3934/math.2020133

[10] Vyas, P., Soni, S. (2018). Entropy analysis for boundary layer flow due to a point sink at the vertex of the cone. Acta Technica, 63(2): 143-156.

[11] Srivastava, N., Vyas, P., Soni, S. (2017). Entropy generation analysis for oscillatory flow in a vertical channel filled with Porous Medium. IEEE International Conference on Recent Advances and Innovations in Engineering (ICRAIE-2016), Jaipur, India, pp. 23-25. https://doi.org/10.1109/ICRAIE.2016.7939542

[12] Vyas, P., Khan, S. (2016). Entropy analysis for MHD dissipative Casson fluid flow in porous medium due to stretching cylinder. Acta Technica, 61(3): 299-315.

[13] Vyas, P. Srivastava, N. (2015). Entropy analysis of generalized MHD Couette flow inside a composite duct with asymmetric convective cooling. Arab. J. Sci. Eng., 40: 603-614. https://doi.org/10.1007/s13369-014-1562-0

[14] Vyas, P., Srivastava, N. (2013). Radiation effects on dissipative magenetohydrodynamic Couette flow in a composite channel. Z. Naturforsch, 68: 554-566. https://doi.org/10.5560/ZNA.2013-0038

[15] Vyas, P., Ranjan, A. (2015). Entropy analysis for MHD generalised Couette flow in a composite duct. Journal of Industrial Mathematics, 2015. https://doi.org/10.1155/2015/895046

[16] Chinyoka, T., Mankinde, O.D. (2013). Analysis of entropy generation rate in an unsteady porous channel flow with Navier slip and convective cooling. Entropy, 15(6): 2081-2099. https://doi.org/10.3390/e15062081 
[17] Yazdi, H.M., Abdullah, S., Hashim, I., Sopian, K. (2013). Reducing entropy generation in MHD fluid flow over open parallel microchannels embedded in a micropatterned permeable surface. Entropy, 15(11): 4822-4843. https://doi.org/10.3390/e15114822

[18] Ibanez, G., Lopez, A., Pantoja, J., Moreira, J., Reyes, J.A. (2013). Optimum slip flow based on the minimization of entropy generation in parallel plate microchannels. Energy, 50: 143-149. https://doi.org/10.1016/j.energy.2012.11.036

[19] Makinde, O.D., Eegunjobi, A.S. (2013). Effects of convective heating on entropy generation rate in a channel with permeable walls. Entropy, 15(1): 220-233. https://doi.org/10.3390/e15010220

[20] Vyas, P., Srivastava, N. (2014). Radiation MHD compressible Couette flow in a parallel channel with a naturally permeable wall. Therm. Sci.,18(Suppl.2): S573-S585. https://doi.org/10.2298/TSCI120828099V

[21] Ibanez, G., Lopez, A., Pantoja, J., Moreira, J. (2014). Combined effects of uniform heat flux boundary conditions and hydrodynamic slip on entropy generation in a microchannel. Int. J. Heat Mass Transfer, 73: 201 206.

https://doi.org/10.1016/j.ijheatmasstransfer.2014.02.007

[22] Lopez de haro, M., Cuevas, S., Beltran, A. (2014). Heat transfer and entropy generation in the parallel plate flow of a power-law fluid with asymmetric convective cooling. Energy, 66: 750-756. https://doi.org/10.1016/j.energy.2013.12.046

[23] Ibanez, G. (2015). Entropy generation in MHD porous channel with hydrodynamic slip and convective boundary conditions. Int. J. Heat Mass Transfer, 80: 274 280.

https://doi.org/10.1016/j.ijheatmasstransfer.2014.09.025

[24] Srinivasacharya, D., Bindu, K.H. (2016). Entropy generation in a micropolar fluid flow through an inclined channel. Alexandria Engineering Journal, 55(2): 973-982. https://doi.org/10.1016/j.aej.2016.02.027

[25] Kareem, S.O., Adesanya, S.O., Vincent, U.E. (2016). Second law analysis for hydromagnetic couplestress fluid flow through a porous channel. Alexandria Engineering Journal, 55(2): 925-931. https://doi.org/10.1016/j.aej.2016.02.020

[26] Ajibade, A.O., Onoja, T.U. (2017). Entropy generation and irreversibility analysis due to steady mixed convection flow in a vertical porous channel, International Journal of Heat and Technology, 35(3): 433-446. https://doi.org/10.18280/ijht.350301

[27] Ogunseye, H.A., Sibanda, P. (2019). A mathematical model for entropy generation in a Powell-Eyring nanofluid flow in a porous channel. Heliyon, 5(5): e01662. https://doi.org/10.1016/j.heliyon.2019.e01662

[28] Ibáñez, G., López, A., López, I., Pantoja, J., Moreira, J., Lastres, O. (2019). Optimization of MHD nanofluid flow in a vertical microchannel with a porous medium, nonlinear radiation heat flux, slip flow and convectiveradiative boundary conditions. J Therm Anal Calorim. 135: 3401-3420. https://doi.org/10.1007/s10973-0187558-3

[29] Saeed, A., Shah, Z., Dawar, A., Islam, S., Khan, W., Idrees, M. (2019). Entropy generation in MHD flow of carbon nanotubes in a rotating channel with four different types of molecular liquids. International Journal of Heat and Technology, 37(2): 509-519. https://doi.org/10.18280/ijht.370218

[30] Sindhu, S., Gireesha, B.J. (2020). Flow of colloidal suspension and irreversibility analysis with aggregation kinematics of nanoparticles in a microchannel. Applied Mathematics and Mechanics, 41: 1671-1684. https://doi.org/10.1007/s10483-020-2669-9

[31] Vyas, P., Yadav, K. (2020). EGA for a convective regime over a vertical cylinder stretching linearly. Applied Mathematics and Nonlinear Sciences, 2020: 1-12. https://doi.org/10.2478/amns.2020.2.00058

[32] Modaledi, R.L., Makinde, O.D. (2021). Entropy generation analysis in a microchannel Poiseuille flows of nanofluid with nanoparticles injection and variable properties. J Therm Anal Calorim., 143: 1855-1865. https://doi.org/10.1007/s10973-020-09919-x

[33] Eegunjobi, A.S., Makinde, O.D. (2020). Inherent irreversibility in a reactive and radiating magnetic nanoliquid film along a slippery inclined heated surface with convective cooling. Heat Transfer, 49: 1533-1553. https://doi.org/10.1002/htj.21675

[34] Rosales, I.G., Duharte, G.I., López Grijalva, A., Lastres Danguillecourt, O., Reyes-Nava, J. (2020). Entropy generation minimization and nonlinear heat transport in MHD flow of a couple stress nanofluid through an inclined permeable channel with a porous medium, thermal radiation and slip. Heat Transfer, 49: 4878-4906. https://doi.org/10.1002/htj.21858

[35] Vyas P., Khan, S.G. (2021). Micropolar couple stress thermofludics and entropy in Forchheimer channel. Heat Transfer, 2021: 1-35. https://doi.org/10.1002/htj.22080

[36] Roja, A., Gireesha, B.J., Nagaraja, B. (2021). Irreversibility investigation of Casson fluid flow in an inclined channel subject to a Darcy-Forchheimer porous medium: a numerical study. Appl. Math. Mech.-Engl. Ed. 42: 95-108. https://doi.org/10.1007/s10483-021-2681-9

[37] Ghai, M.L., Jacob, M. (1950). Local Coefficient of Heat Transfer on Fins, ASME Paper.

[38] Lau, W., Tan, C.W. (1973). Errors in one-dimensional heat transfer analysis in straight and annular fins. ASME J. Heat Transfer, 95: 549-551. https://doi.org/10.1115/1.3450110

[39] Laor, K., Kalman, H. (1996). Performance and optimum dimensions of different cooling fins with a temperature dependent heat transfer coefficient. Int. J. Heat Mass Transfer, $39(9)$ : https://doi.org/10.1016/0017-9310(95)00296-0

[40] Unal, H.C. (1985). Determination of the temperature distribution in an extended surface with a nonuniform heat transfer coefficient. Int. J. Heat Mass Transfer, 28(12): 2270-2284. https://doi.org/10.1016/00179310(85)90046-8

[41] Sen, A.K., Trinh, S. (1986). An exact solution for the rate of heat transfer from rectangular fin governed by a power law-type temperature dependence. Trans. ASME J. Heat Transfer 108(2): 457-459. https://doi.org/10.1115/1.3246946

[42] Vyas, P., Ranjan, A. (2015). Entropy analysis of radiative MHD forced convection flow with weakly temperature dependent convection coefficient in porous medium channel. Acta Technica, 60(1): 1-14.

[43] Beavers, G.S., Joseph, D.D. (1967). Boundary conditions at a naturally permeable wall. J. Fluid Mech., 30(1): $197-$ 207. https://doi.org/10.1017/S0022112067001375

[44] Saffman, P.G. (1971). On the boundary condition at the 
surface of a porous medium. Stud. Appl. Math., 50: 93 101. https://doi.org/10.1002/sapm197150293

[45] Dagan, G. (1979). The generalization of Darcy' law for nonuniform flows. Water Resource Research, 15(1): 1-7. https://doi.org/10.1029/WR015i001p00001

[46] Taylor, G.I. (1971). A model for boundary conditions of a porous material Part 1, J. Fluid Mech., 49: 319-326. https://doi.org/10.1017/S0022112071002088

[47] Richardson, S. (1971). A model for the boundary condition of a porous material-Part 2. J. of Fluid Mech., 49(2):

$327-336$

https://doi.org/10.1017/S002211207100209X
[48] Larson, R.E., Higdon, J.J.L. (1986). Microscopic flow near the surface of two-dimensional porous media - Part 1 Axial flow. J. Fluid Mech., 166: 449-472. https://doi.org/10.1017/S0022112086000228

[49] Larson, R.E., Higdon, J.J.L. (1987). Microscopic flow near the surface of two-dimensional porous media - Part 2 transverse flow. J. Fluid Mech., 178: 119-136. https://doi.org/10.1017/S0022112087001149

[50] Sahraoui, M., Kaviany, M. (1992). Slip and no-slip velocity boundary conditions at the interface of porous plain media. Int. J. Heat Mass Transfer, 35(4): 927-943. https://doi.org/10.1016/0017-9310(92)90258-T 